\section{QUETES IDENTITAIRES DANS NOTRE MODERNITE}

\section{Buscas Identitárias em Nossa Modernidade}

Identity Searches in Our Modernity

Búsquedas de Identidades en Nuestra Modernidad
Biopolítica e Subjetividades Contemporâneas

\title{
Résumé
}

L'aliénation du sujet à la civilisation technoscientifique et à l'économie de marché se traduit par la promotion de l'égo et de ses exploits imaginaires, une souffrance commune devant les nouveaux modes de vie. L'affirmation décisive du discours de la science annonce un changement radical dans les possibilités de subjectivation, l'émergence de nouvelles subjectivités et surtout une modification majeure dans le rapport au corps et à l'identité.

Mots-clés: discours; technoscience; corps; subjectivité; identité.

\section{Resumo}

A alienação do sujeito na civilização tecnocientífica e na economia de mercado se traduz pela promoção do ego e de suas façanhas imaginárias, um sofrimento comum diante dos novos modos de vida. A afirmação decisiva do discurso da ciência anuncia uma mudança radical nas possibilidades de subjetivação, a emergência de novas subjetividades e sobretudo uma grande mudança em relação ao corpo e a identidade.

Palavras-chave: discurso; tecnociência; corpo; subjetividade; identidade.

\section{Abstract}

The subject alienation in techno-scientific civilization and market economy is translated by the promotion of the ego and its imaginary feats, a common suffering in the face of new ways of life. The decisive statement of the science discourse announces a radical change in the possibilities of subjectivity, the emergence of new subjectivities and above all a big change concerning the body and identity.

Keywords: speech; technoscience; body; subjectivity; identity.

\section{Resumen}

La alienación del sujeto en la civilización industrial y en la economía de mercado se traduce en la promoción del ego y sus hazañas imaginarias, un sufrimiento común delante de los nuevos modos de vida. La afirmación decisiva del discurso de la ciencia anuncia un cambio radical en las posibilidades de tornarse sujeto, la emergencia de nuevas subjetividades $y$, sobre todo un gran cambio en relación al cuerpo y a la identidad.

Palabras clave: discursos; industrial; cuerpo; subjetividad; identidad.

\section{Jean-Luc Gaspard ${ }^{(1)}$}

\author{
1) Maître de Conférences \\ en Psychopathologie, Psychanalyste, \\ Directeur de la composante "Recherches \\ en Psychopathologie: pratiques et champs \\ spécifiques".
}


La montée en puissance de problématiques socioéconomiques, politiques et/ou religieuses associées à la question identitaire ne peut laisser indifférents les chercheurs comme les cliniciens. Dans ces temps présents où se conjuguent, de manière parfois conflictuelle, maligne ou violente, localisme et mondialisation, "l'inquiétante étrangeté" ne paraît désormais plus renvoyer à l'altérité dans ses formes traditionnelles mais au voisinage, à cet autre qui n'est pas de notre monde, de nos traditions et culture, voire tout simplement de notre mode de vie. Et c'est dans cette voie que les discours fondamentalistes ou passéistes célébrant le recours à quelque lointain passé mythique, national ou religieux viennent ranimer les fantasmes identitaires au travers de catégories idéologiquement revivifiées: nation, peuple, classe, race, ethnie, etc. A partir de ce constat, les sociologues et les philosophes contemporains inscrivent ce mouvement dans de nouvelles modalités historiques de fabrication de l'identité. Comme le souligne Kaufmann (2014, p. 61), malgré la réflexivité critique et la créativité des singularités personnelles, "tous les fondamentalismes (communautaristes, nationaux, religieux ou raciaux) se renforcent actuellement. Les dérives identitaires sont une véritable bombe à retardement". Certes la notion d'identité apparaît comme une notion aux contours flous et aux définitions incertaines. Ce n'est que progressivement que celle-ci a pris consistance dans le champ des sciences humaines pour s' imposer comme une question voire une problématique dominante de notre époque. A contrecourant de toutes les thèses substantialistes ou essentialistes qui fantasment une identité fixe et stable, l'identité doit être située du côté de la subjectivité et de la production de sens. Mieux, l'identité est avant tout processus: "l'identité est ce qui ferme le sens et crée les conditions de l'action" (Kaufmann, 2014, p 17).

Selon l'hypothèse sociologique, la rupture historique entre sociétés holistes et sociétés individualistes (Dumont, 1983) porte en germe la place centrale que le processus identitaire va occuper dans notre modernité. En effet, plus la société se présente comme ouverte et plus les individus sont contraints de choisir (ou non) d'appartenir à un groupe, de participer à la production de nouvelles normes, là où les cadres collectifs (et généralement religieux) donnaient historiquement des réponses communes, une orientation et un sens pour chacun sur ce qu'il convenait de vivre. Or si l'on admet que la science moderne s'est bâtie sur un véritable "oubli de l'être", si nous sommes dans un temps où l'être est en souffrance, alors les conséquences de cette situation inédite ne peuvent être que radicales au niveau de ce qui devient une crise des identités. Le franchissement de la clôture que constituait la métaphysique, la suspension de la vérité ontologique, le pouvoir de manipulation de la nature dépassent ainsi toute volonté chez le commun de construction de sens de l'existence. Discours de la science avec sa profusion d'instruments et de gadgets technologiques mis sur le marché, nouveaux modes de jouissance que ce premier trouve ainsi à introduire, réel du monde toujours plus prégnant qui impacte les sujets, promotion de la forclusion du sujet: nous n'en avons pas fini de décliner les effets délétères produits sur les corps traversés par le langage, les situations d'inquiétante étrangeté dans la vie sociale comme l'affrontement entre subjectivités. A la modernité "organisée" de la première moitié du XX ème siècle où les individus restent dans très large mesure socialement construits par les institutions, les classes sociales d'appartenance, les communautés de vie ou de travail va succéder plus particulièrement dans nos démocraties occidentales une seconde phase où les individus cherchent à s'émanciper tout en se soumettant toujours plus aux nouveaux dogmes du discours de la science. Là où autrefois les sociétés étaient locales, bien définies et structurées à partir de rituels religieux ou d'initiation, les modes actuels de constitution du corps et du rapport à la sexualité en viennent à concentrer pour chacun la possibilité d'un "accès à sa propre intelligibilité, à la totalité de son corps, à son identité" (Foucault, 1976, p. 205). Ce mouvement de revendication individualiste, ce souci contemporain de soi dont les démocraties modernes font promotion semble agir comme un leurre face à un malaise dans la culture toujours aussi présent et face à une domination des discours toujours plus prégnante. Afin de penser le contexte social qui est le cadre des nouveaux modes de production de subjectivités, des nouveaux scénarios de production des symptômes dans la culture, le présent article se propose à partir des travaux de Foucault et de l'enseignement de Lacan d'analyser les avatars des modes contemporains de subjectivation et de constitution de l'identité ainsi que la place qu'y occupe désormais le corps.

\section{La Problématique de L'identité chez Foucault}

Nous n'en sommes pas surpris. C'est sur la question du mode de vie gay que Foucault, dans le cadre de son enseignement, va revenir sur la question identitaire. Prenant acte du fait que les années 1970 ont vu l'émergence d'une libéralisation des mœurs et, pour l'individu, la revendication "d'avoir la possibilité - et le droit- de choisir sa sexualité" (Foucault, 1994b, p. 736), Foucault estime que cette situation nouvelle dans nos sociétés occidentales ne sera stabilisée que par la création de nouvelles formes d'expression, de nouveaux rapports et liens dans la société, l'art, la culture qui s'instaureront au travers des choix sexuels, éthiques et politiques. Et de poursuivre: "nous devons non seulement nous défendre, mais aussi nous affirmer, et nous affirmer non seulement en tant qu'identité, mais en tant que force créatrice" (Foucault, 1994b, p. 736). Mais souhaiter l'avènement d'autres types de créations culturelles ramène 
Foucault aux deux problèmes majeurs que pose l'identité: le premier d'ordre collectif serait de résumer la culture gay à un "entre soi" réducteur. Par exemple, Foucault met en doute le fait que "la meilleure forme de création littéraire que l'on puisse attendre des homosexuels soit les romans homosexuels" (Foucault, 1994b, p. 737). Dans cette tentation identitaire de "donner corps" et de tenter par tous les moyens de défendre comme de faire la promotion d'un groupe ou d'une communauté, le risque est de participer à la logique de ségrégation en considérant l'identité acquise ou revendiquée comme une règle éthique universelle. Le second problème en découle et renvoie à la logique individuelle lorsque l'identité (fût-elle sexuelle) sert de fil, de vecteur unique pour tenter d'ordonner l'existence. Certes, le fait d'être "unique" oblige chacun d'entre nous à se situer par rapport à la question de l'identité mais celleci - insiste Foucault - doit rester "un jeu, un procédé pour favoriser des rapports, des rapports sociaux et des rapports de plaisir sexuel qui créeront de nouvelles amitiés" (Foucault, 1994b, p. 739). A défaut, l'identité affirmée ne peut que réintroduire une dissonance au niveau individuel entre souci d'appartenance à une communauté et aspiration à l'autonomie personnelle.

La question identitaire n'est qu'esquissée chez Foucault et passe par une conception dynamique, processuelle. Au même titre que l'émergence (par des actions critiques de résistance) de nouvelles relations humaines, l'émergence de nouvelles identités vient aussi servir de point d'appui pour articuler théoriquement dans une seconde partie de son enseignement les notions de "gouvernementalité" et ce qu'il définira comme relevant des "techniques de soi": D'une part le contrôle des individus par la société dépasse les champs de la conscience et de l'idéologie pour relever d'une socialisation du corps. "Pour la société capitaliste, c'est le biopolitique qui importait avant tout, le biologique, le somatique, le corporel. Le corps est une réalité biopolitique" (Foucault, 1994a, p. 210). Et au croisement des populations, du "Bio-pouvoir" et de la gestion de la vie, des dispositifs de pouvoir et des individus un savoir de la sexualité est convoqué. Le sexe devient ainsi "le point imaginaire par lequel chacun doit passer pour avoir accès à sa propre intelligibilité, à la totalité de son corps, à son identité" (Foucault,1976, p. 188).

Sur un autre plan, dans son cours de 1980-1981, Foucault souligne l'importance des techniques de soi en regard de la fabrique de l'identité. Celles-ci sont définies comme:

(...) les procédures, comme il en existe sans doute dans toute civilisation, qui sont proposées ou prescrites aux individus pour fixer leur identité, la maintenir ou la transformer en fonction d'un certain nombre de fins, et ce grâce à des rapports de maîtrise de soi sur soi ou de connaissance de soi par soi. (Foucault, 2014, p. 213)
On peut mesurer l'importance de l'étude du souci et des techniques de soi, bref du gouvernement de soi par soi "dans son articulation avec les rapports d'autrui (comme on le trouve dans la pédagogie, les conseils de conduite, la direction spirituelle, la prescription des modèles de vie, etc..)" (Foucault, 2014, p. 217) pour saisir ce qu'il en est à une époque donnée de la subjectivité.

Même si Foucault ne fait qu'esquisser une réflexion sur l'évolution de la question identitaire dans notre modernité, nous voyons bien que l'intérêt qu'il porte sur cette question relève pour ses lecteurs de trois registres indissociables de son œuvre: celui du philosophe, du militant politique et enfin du défenseur de la cause et du mode de vie homosexuel. Foucault insiste sur le fait que les modes de subjectivation sont indissociables d'une herméneutique de soi et sont donc des techniques de soi éthiques, des morales orientées vers l'éthique (Foucault, 1984, p. 37). Il maintient cependant la distinction entre le souci de soi dans l'antiquité et celui qui a cours dans notre actualité. D'un côté, un sujet antique constitué par des pratiques de soi et de l'autre, un sujet moderne fondateur de nouvelles pratiques de connaissances. Abordant ce que Baudelaire reconnaît comme du dandysme, Foucault souligne qu': “être moderne, ce n'est pas s'accepter soi-même tel qu'on est dans le flux de moments qui passent; c'est se prendre soi-même comme objet d'une élaboration complexe et dure" (Foucault, 1994b, p. 570) pour conclure par cette affirmation: "L'homme moderne est celui qui cherche à s'inventer lui-même" (Foucault, 1994b, p. 571). Il s'agit donc pour chaque sujet de prendre position de manière critique sur les événements qui l'ont amené à se constituer et à se reconnaître comme sujet de ce qu'il fait, pense ou dit (Foucault, 1994b, p. 574) dans une constitution de soi-même comme sujet autonome.

Ainsi, dans les complexes rapports sociaux ou de plaisir sexuel, par la possibilité d'utiliser son corps au travers d'expériences (exemple des prises de drogues, du sport extrême, etc.), de solliciter de nouvelles amitiés, Foucault estime qu'il est possible de dépasser une représentation figée de l'identité et de favoriser, voire revendiquer "des rapports de différenciation, de création, d'innovation" Car dit-il: il est "très fastidieux d'être toujours le même" (Foucault, 1994c, p. 718). Face aux rapports de pouvoir qui ont tendance à se rigidifier, Foucault insiste sur la fluidité des sous-cultures et l'importance des rapports stratégiques seuls capables de permettre aux individus non pas de s'acharner à renverser un pouvoir d'un état moderne toujours plus totalitaire mais de mettre en question l'identité corrélative au pouvoir d'Etat, identité faite tout autant de la promotion individualisante que d'une dépendance toujours plus exacerbée à l'endroit du "Bio-pouvoir". 


\section{Identité(s), Identification(s) et Modes D'être du Sujet}

Pour étudier le lien entre l'émergence de nouvelles subjectivités, quêtes identitaires et discours dominants en référence avec l'apport théorique de Lacan, il est nécessaire de faire quelques précisions théoriques. Tout d'abord, rappelons que la notion d' "identité" n'est pas un concept de la psychanalyse mais doit être rattachée à la catégorie d'identification. Freud avait distingué trois modalités d'identification liées à l'Autre et qui ne répondent pas à l'existence d'une identité propre au sujet: L'identification primordiale au père, celle d'avant tout lien objectal, préœdipienne, l'identification qui s'inscrit dans le registre œdipien et se fonde sur un trait particulier emprunté à l'objet investi par la libido et enfin l'identification hystérique, c'est-à-dire une identification au rapport qu'entretient cet autre à l'égard du désir. Lacan, quant à lui, interprètera cette première identification freudienne comme incorporation signifiante d'où procède le désir, rattachera la seconde au fameux einziger Zug ou trait unaire. Quand à la troisième, elle prendra appui sur le discours de l'hystérique comme lien social fondé sur un désir de désir. Enfin, Lacan dégagera à la fin de la cure une autre modalité d'identification: l'identification au symptôme renvoyant à l'identité la plus singulière et qui toucherait à l'être du sujet.

Dans son Séminaire IX, "L’identification" (Lacan, 1961-1962), lors de la séance leçon du 15 novembre 1961, Lacan souligne que l'identification permet à "chercher le sens de toute identité, au cœur de ce qui se désigne par une sorte de redoublement de moi-même" là où "s'engouffrent, après le moi, le toi, le lui, le elle, le eux, le nous, le vous". Bref, si l'identité est toujours singulière, elle se fonde sur une pluralisation d'identifications du fait de l'aliénation symbolique et renvoie aussi à la captation imaginaire du sujet à l'Autre. Ainsi la question identitaire renvoie à la fois à la structure subjective (névrose, psychose, perversion) mais aussi au(x) discours comme effet d'après-coup de ce qu'il en advient de l'Autre (social, familial) et des institutions (au premier rang de laquelle figure celle du langage), qui, à une période donnée, marquent "dans le culturel le recoupement du biologique et du social" (Lacan, 1966a, p. 184).

Dès 1953, dans son étude des rapports dans le sujet de la parole et du langage, Lacan pointait déjà le fait d'un "sujet qui perd son sens dans les objectivations du discours" (Lacan, 1966b, p. 281). Mais c'est un peu plus tard que le courant lacanien s'est intéressé à la question du lien éventuel entre la subjectivité et le malaise de la civilisation. Comme le relève Sauret (2000, p. 182), "la subjectivité, Lacan l'identifie à l'articulation de quatre termes: savoir, vérité, sujet et rapport à l'Autre. De sorte que nous pourrions soupçonner que la 'subjectivité de notre époque' correspond à un mode particulier de leur articulation”. Lorsque Lacan conseille à tout psychanalyste "qui ne peut rejoindre à son horizon la subjectivité de son époque" (Lacan, 1966b, p. 321) de renoncer à la pratique, ce n'est donc pas en tant que sujet "tout-sachant" mais bien plutôt en tant que clinicien capable d'accueillir (dans une mise en fonction du "sujet supposé savoir") tous les évènements symptomatiques, toutes les crises et cristallisations qui viennent à sa rencontre. En effet, la place du sujet supposé savoir est cette

(...) position inaugurale de l'acte psychanalytique qui consiste à jouer sur quelque chose que l'acte justement va démentir (...) Le psychanalyste n'est sans doute pas le seul à connaître la radicale division de sujet supposé savoir, mais il est particulièrement bien placé pour en prendre la mesure. (Lacan, 1966b, p. 183)

Aussi, en portant toute son attention sur les territoires de la jouissance, dans la dernière partie de son enseignement, Lacan a cherché à aborder cette masse hétérogène de subjectivités et toutes les formes plus ou moins radicales d'instrumentation objectivante promues par les dispositifs et les nouvelles technologies. Comme il le faisait fort justement remarquer à son auditoire: "Vous êtes désormais, infiniment plus loin que vous le pensez, les sujets des instruments qui, du microscope jusqu'à la radio-télévision, deviennent des éléments de votre existence“ (Lacan, 1975, p. 76). Cette situation inédite où se conjuguent et s'opposent plusieurs formes de modernité (Attali, 2013) nous incite désormais à réfléchir au devenir de la logique structurale partiellement entamée qui préside, d'une part, à la construction du sujet et, d'autre part, qui sert de fondement aux relations interindividuelles.

Sur ce point, Lacan a rappelé l'impact de cette collusion inédite entre discours du capitaliste et discours de la science. Le discours dit du capitaliste, dans la formalisation qu'il propose à Milan en 1972, opère en effet une véritable torsion en regard du discours du maître en consacrant la rupture du lien entre le sujet et le savoir et entre la vérité et la jouissance. Cette version pousse le sujet de ce discours à s'inscrire dans la logique du "self made man", tout en étant "prolétarisé", soumis à l'appétit cumulatif des objets et la mise en jeu toujours plus effrénée de ses propres ressources. Conséquences: le refus du manque, voire de l'altérité mais aussi la multiplication parallèle des techniques de corps venant répondre aux vertiges comme à la souffrance de l'être (Gaspard, 2014). Sur un autre plan, les idéologies scientistes actuelles s'inscrivent dans la promesse fétichiste et intégriste d'un monde de complétude. Un monde dans lequel il serait possible de dompter la pulsion et de domestiquer la jouissance pour être en harmonie avec son corps. Au droit de pouvoir jouir de son corps (Gaspard \& Doucet, 2009) semble s'associer une fiction contemporaine; celle d'être à l'image de son organisme. Cette croyance est alimentée par un courant neurobiologique qui, convaincu que l'être s'y avère réductible, tendrait à vouloir identifier 
chacun à partir de marqueurs biologiques, somatiques et génétiques. Dans un tel contexte, parler de "nouveaux" symptômes relève pour les cliniciens d'aujourd'hui de la confrontation à une prédominance du réel et de la jouissance. Ce qui nous écarte de la version symbolique du symptôme comme recel de vérité inconsciente (Freud) ou du symptôme lacanien comme étant la solution la plus singulière du sujet lui permettant de s'inscrire dans le lien social et de se défendre de la domination (voire voracité) de l'Autre (Sauret, 2008).

\section{Dérives Identitaires: Entre Intégrisme et Fanatisme}

La présentation du nouveau malaise dans la civilisation permet-elle de penser les dérives identitaires observées dans nos sociétés? Lacan a insisté sur le procès de production de chaque discours (qu'ils s'agissent d'évènements discursifs ou de nouvelles formes de subjectivité) et la formalisation qu'il nous propose dans son enseignement a le mérite de révéler (par l'introduction de la catégorie de jouissance) comment trouvent à se nouer les dimensions politique et économique (Gaspard, 2010). Historiquement chaque discours, "à prendre comme lien social, fondé sur le langage" (Lacan, 1975, p. 21), doit permettre aux sujets de trouver à s'y loger tout en parvenant à régler leurs propres rapports à la jouissance tout en permettant d'en prélever ou d'en récupérer des bribes (que Lacan appelle "plus de jouir"). C'est un dispositif de régulation de la jouissance qui ne fait qu'encadrer, légitimer, officialiser des pratiques normatives mais aussi des pratiques d'exclusion et de ségrégation (Sauret, 2000, p. 121). Sous l'hégémonie conjointe de la loi du marché et du discours de la science, résulte tout d'abord une forme d'obsolescence du Politique (défaite du communisme, affaiblissement des démocraties, dissolution d'états) notamment face à l'émergence progressive de nouvelles entités économiques (multinationales, mafias, oligarchies). Ce glissement moderne, à savoir la domination de l'économique sur le politique, prétend évacuer la dimension de l'impossible. Confrontés à de nouvelles économies de jouissance (Melman, 2005; Melman \& Lebrun, 2009), chaque discours actuel se voit ébranlé au niveau de ses assisses symboliques comme de son économie interne et, de facto, se trouve toujours plus enclin à suivre une pente ségrégative, dans un procès de production en quelque sorte "forcée" d'une subjectivité désormais tournée vers les nouvelles donnes idéologiques et socio-économiques (adaptabilité, compétitivité, concurrence exacerbée, etc.).

Parallèlement, la constitution du village global via internet, la multiplication de vagues migratoires souvent forcées (guerres civiles, réchauffement climatique), les modes contemporains de consommation conduisent à un nivellement des différences. Nombre de nos contemporains se trouvent subjectivement "exilés" (disparition progressive des traditions, éloignement des racines, terroirs, us et coutumes). Le rapport de chaque sujet au savoir (roman familial, mythe des origines) s'en trouve ainsi fortement modifié alors même que s'appauvrissent les ressources de la structure (névrose, psychose ou perversion) comme les montages symboliques des discours. Face à un réel potentiellement désarrimé des défilés de la vérité, sont ainsi exacerbés la quête de sens et le rejet de l'hétéronomie.

Par ailleurs les impasses que l'on rencontre dans la constitution identitaire au niveau individuel peuvent venir à se collectiviser en identités collectives comme autant de points de fixation du narcissisme de groupe. On retrouve le paradoxe de la problématique identitaire qui oscille systématiquement entre aliénation (cristallisation des identifications) et déprise (des identifications normatives). Ainsi peut-on comprendre les revendications identitaires actuelles dans une double tension entre protestation et affirmation (qu'il s'agisse pour exemple de la question du genre et des minorités sexuelles qui conduisent à un relativisme et à une fragmentation sans précédent des pratiques sexuelles ou, à l'inverse, des formes défensives les plus diverses, parfois réactionnaires devant ce qui est considéré comme la dissolution de l'ordre patriarcal, d'un héritage régional ou sociétal). C'est dans cette veine que Lacan "prophétisait" -à contre-courant de ce qui était pensé à son époque - le triomphe de la religion:

Le réel, pour peu que la science y mette du sien, va s'étendre, et la religion aura là beaucoup de raisons encore d'apaiser les cœurs. La science, c'est du nouveau, et elle introduira des tas de choses bouleversantes dans la vie de chacun. (...) Et la religion va donner du sens aux épreuves les plus curieuses, celles dont les savants eux-mêmes commencent justement à avoir un petit bout d'angoisse. (Lacan, 2005, pp. 79-80)

L'on constate en effet combien les identités religieuses (pentecôtisme et églises évangélistes, fondamentalismes musulman, bouddhiste, hindouiste, etc.) peuvent servir de support topologique à l'identification des sujets. Elles procurent un certain apaisement aux individus par les liens communautaires qu'elles tissent et viennent répondre à l'angoisse devant les risques de désarrimage subjectif ou collectif. Or si, par ce retour au religieux, il s'agit de donner du sens à la jouissance, c'est loin d'être à tout coup un progrès mais bien souvent une régression.

En effet, c'est une volonté de pureté (Lévy, 1994; Bruckner, 1995) qui anime nombre d'intégrismes (religieux, écologiques, etc.). Dans les doxas fondamentalistes, il n'est reconnu aucune faute, aucun péché originel, aucune grande catastrophe ayant pu troubler le monde parfait et harmonieux de l'origine, là où l'ordre régnait sur le monde et la communauté. C'est cette innocence affirmée aux temps 
des fondements et cette perfection initiale qui auraient été corrompues dans l'après-coup et qui doivent être retrouvées. Ce projet, sous de multiples variantes, alimente toutes les formules de primitivisme, de naturalisme, tous les espoirs de retour aux commencements mythiques du groupe, du peuple ou de la nature. Ces fantasmes d'unité ou d'unanimisme permettent à celles et ceux qui s'en soutiennent de rêver d'une société ou d'un monde débarrassé de ses scories et de toutes impuretés. Dans le fondamentalisme, seul le Texte fondateur posé comme absolu permet de prendre appui pour interpréter comment revenir aux sources de la Vérité. C'est pourquoi, très souvent, dans une logique paranoïde, l'altérité qu'elle soit à l'intérieur du corps social ou de voisinage devient menaçante. La logique d'épuration et d'exclusion qui en découle, déjà présente dans les idéologies léninistes (société sans classes) et national-socialistes (le Volk allemand) du XXème siècle, est de nouveau recyclée, réactualisée et prend corps dans des génocides ethniques comme dans la chasse aux minorités religieuses, immigrés, homosexuels, etc.., bref contre tout ce qui est censé altérer le retour à une pureté originelle.

Là où l'intégrisme promeut un retour aux sources notamment dans les pratiques religieuses, le fanatisme se caractérise par une inversion des valeurs permettant à la négativité de prendre sens, que ce soit comme moyen ou comme fin. Cette inversion conduit à penser que c'est sur les ruines du présent (ou d'un passé maudit) que peuvent revenir les fleurs d'un passé mythique ou se déployer le futur de l'Idéal. Le fanatisme s'inscrit alors dans le registre de la déliaison pulsionnelle.

Il n'est pas de fanatique qui ne soit au service de Thanatos, que la destructivité soit tournée vers l'autre ou qu'elle soit tournée vers lui-même. Le fanatisme, au-delà de ses légitimations partielles ou ponctuelles, est avant tout un culte du sacrifice. (Chouvier, 2009, p. 10)

Cette reconquête de l'identité procure là encore certains bénéfices: elle substitue au commandement erratique d'un objet de jouissance le signifiant-maître qui pourra éventuellement pousser au sacrifice suprême, celui de la vie. Surtout, elle conduit à une stase du sens qui vient figer l'identification. Mais cette tentative de métaphorisation (qui vire au signe sans équivoque) ouvre trop souvent la voie à toutes les logiques persécutrices voire extrémistes ou terroristes.

\section{Conclusion}

Comme nous pouvons le constater, cette question de l'identité (et en sous-main celle des identifications du sujet) apparaît déterminante pour penser la modification actuelle des liens sociaux contemporains et cruciale pour la clinique d'aujourd'hui (Thibierge, 2007) puisqu'elle convoque le psychanalyste dans son engagement à des enjeux éthiques et une logique de l'acte. Face aux mutations géopolitiques du nouveau siècle, à la chute des idéologies du XXème siècle, à la "mise en épave" de l'inconscient et surtout à de nouveaux modes d'organisation socio-économique ou familiale, l'émergence d'un patchwork identitaire et identificatoire n'est pas une surprise. Dans la littérature psychanalytique d'orientation lacanienne, il existe un accord plutôt général concernant les effets du discours de la science sur la constitution du sujet. On y parle d'une forclusion (ou rejet) du sujet par évitement notamment de l'interrogation sur l'être comme sur la mort. Cette forclusion, ce rejet se traduit par la méconnaissance du sens particulier pour chacun de sa vie au profit de la promotion de l'ego et de ses exploits imaginaires (Lipovetsky, 1983; Lasch, 2006). On peut repérer les conséquences subjectives de cette économie actuelle de la normalisation au travers de la variété des réponses individuelles (adhésion, soumission, résistance, objection, opposition) de mise en jeu du corps, de nouveaux modes de jouir ou de nouvelles manières pour les sujets de prendre position vis-à-vis du savoir, du pouvoir et du sexe. Désormais au service du discours du capitaliste, cette version mondialisée de l'aliénation technoscientifique (Askofaré, 2014) introduit d'un côté les sujets à de nouveaux réels tout en fournissant aux individus de nouvelles modalités orthopédiques ou normatives censées traiter le malaise actuel. Cette recherche d'une suture du réel par les dispositifs (Agamben, 2007) et les outils techniques ou statistiques conduit à représenter l'espace social en chiffres et en lettres, comme matière homogène sans contradictions internes, dans l'attente de l'extraction et de l'accumulation des ressources (humaines). Notre époque est en effet marquée par l'emprise croissante du comptage, de la quantification. La tyrannie du tout-chiffrage - qui n'est pas sans faire écho au nouveau culte de la transparence et à la chute des idéaux - modifie sensiblement les modes d'être du sujet. Les effets psychosociaux des projets de domination $\mathrm{du}$ vivant (intelligence artificielle, procréation assistée, neurosciences, cyborg, etc.) que n'hésitent plus à afficher certaines multinationales du numérique ne peuvent que s'opposer à l'idée d'une société politique conçue comme essentiellement lacunaire. Face à une défaite partielle de la logique d'ordre des discours (Foucault, 1970) et à une fragmentation sans précédent des modalités d'inscription des sujets dans les liens sociaux actuels, répondent en retour et en réaction les quêtes identitaires, les pratiques de ségrégation et les affrontements de subjectivités. En regard du discours de la science, la problématique de l'identité comme le propose Askofaré (2007):

(...) cesse d'être une question de reconnaissance, une question d'articulation ou de nouage de l'image et du nom - question du sens, pourrait-on dire - pour dériver 
vers celle du mot et de la chose - question de la référence cette fois -, du nom et de l'objet, de la correspondance du symbolique et du réel. (Askofaré, 2007, p. 41)

Sur un autre plan, en relation avec la marchandisation des corps promue par le discours du capitaliste, c'est au travers des expérimentations les plus variées du corps (modifications et marquages corporels, sexualités, etc.), voire par un reflux vers le masochisme érogène que certains de nos contemporains cherchent solution à l'insoutenable légèreté de l'être (Kundera, 1984).

\section{References}

Agamben, G. (2007). Qu'est ce qu'un dispositif ? Paris: Rivages Poche, Petite Bibliothèque.

Askofaré, S. (2007). L'identité au temps du discours de la science. Mensuel, (28), 37-42. Recuperee http://www. champlacanienfrance.net/spip.php?article289

Askofaré, S. (2014). D’un discours l'Autre: La science à l'épreuve de la psychanalyse. Toulouse: PUM.

Attali, J. (2013). Histoire de la Modernité: Comment l'humanité pense son avenir. Paris: Robert Laffont.

Bruckner, P. (1995). La tentation de l'innocence. Paris: Grasset.

Chouvier, B. (2009). Les fanatiques. Paris: Odile Jacob.

Dumont, L. (1983). Essais sur l'individualisme. Une perspective anthropologique sur l'idéologie moderne. Paris: Le Seuil.

Foucault, M. (1970). L'ordre du discours. Paris: Gallimard.

Foucault, M. (1976). Histoire de la sexualité 1: La volonté de savoir. Paris: Gallimard.

Foucault, M. (1984). Histoire de la sexualité 2: L'usage des plaisirs. Paris: Gallimard.

Foucault, M. (1994a). La naissance de la médecine sociale (1974). In M. Foucault, Dits et écrits (Vol. 3, pp. 207228). Paris: Gallimard.

Foucault, M. (1994b). Michel Foucault, une interview: sexe, pouvoir et la politique de l'identité (1982). In M. Foucault, Dits et écrits (Vol 4, pp. 735-746). Paris: Gallimard.
Foucault, M. (1994c). L'éthique de soi comme pratique de la liberté (1984). In M. Foucault, Dits et écrits (Vol 4, pp. 708-729). Paris: Gallimard.

Foucault, M. (2014). Subjectivité et vérité. Cours au Collège de France (1980-1981). Paris: Gallimard.

Gaspard, J-L., \& Doucet, C. (2009). Pratiques et usages $d u$ corps dans notre modernité. Toulouse: ERES.

Gaspard, J-L. (2010). Nouveaux symptômes et lien social contemporain. In L. Jodeau-Belle, \& L. Ottavi (Coords.), Les fondamentaux de la psychanalyse lacanienne: Repères épistémologiques, conceptuels et cliniques (pp. 357-372). Rennes: PUR.

Gaspard, J.-L. (2014). La souffrance de l'être: Formes modernes et traitements. Toulouse: ERES.

Kaufmann, J-C. (2014). Identités, la bombe à retardement. Paris: Editions Textuel.

Kundera, M. (1984). L'insoutenable légèreté de l'être. Paris: Gallimard.

Lacan, J. (1966a). Propos sur la causalité psychique (1946). In J. Lacan, Ecrits (pp. 151-193). Paris: Seuil.

Lacan, J. (1966b). Fonction et champ de la parole et du langage en psychanalyse (1953). In J. Lacan, Ecrits (pp. 237-322). Paris: Le Seuil.

Lacan, J. (1961-1962). L’identification, Le Séminaire Livre $I X$. Inédit.

Lacan, J. (1975). Encore, Le Séminaire Livre XX (19721973). Paris: Le Seuil.

Lacan, J. (2005). Le triomphe de la religion (1975). Paris: Le Seuil.

Lasch, C. (2006). La culture du narcissisme: La vie américaine à un âge de déclin des espérances. Paris: Flammarion, Champs.

Lévy, B-H. (1994). La Pureté dangereuse. Paris: Grasset.

Lipovetsky, G. (1983). L'ère du vide: Essais sur l'individualisme contemporain. Paris: Gallimard.

Melman, C. (2005). L'Homme sans gravité: Jouir à tout prix. Toulouse: ERES.

Melman, C., \& Lebrun, J-P. (2009). La nouvelle économie 
psychique: La façon de penser et de jouir aujourd'hui. Toulouse: ERES.

Sauret, M-J. (2000). Psychanalyse et politique: Huit questions de la psychanalyse au politique. Toulouse: PUM. Coll.

Sauret, M-J. (2008). L'effet révolutionnaire du symptôme. Toulouse, ERES. Coll. Humus.

Thibierge, S. (2007). Clinique de l'identité. Paris: PUF.
Endereço para correspondência:

Jean-Luc Gaspard

Endereço: EA 4050, Université Rennes 2, Place Recteur le Moal, 35043 Rennes Cedex, France.

E-mail: jean-luc.gaspard@uhb.fr; jlgaspard@wanadoo.fr 